hep-th/9711165

IHES $/ \mathrm{P} / 97 / 83$

RU-97-95

QMW-PH-97-34

LPTENS 97/56

\title{
D-branes and the Noncommutative Torus
}

\author{
Michael R. Douglas ${ }^{1,2}$ and Chris Hull ${ }^{3,4}$ \\ ${ }^{1}$ Institut des Hautes Études Scientifiques \\ Le Bois-Marie, Bures-sur-Yvette, 91440 France \\ 2 Department of Physics and Astronomy \\ Rutgers University \\ Piscataway, NJ 08855-0849 USA \\ ${ }^{3}$ Physics Department, QMW \\ Mile End Road, London E1 4NS, U.K. \\ ${ }^{4}$ Laboratoire de Physique Théorique, Ecole Normale Supérieure, \\ 24 Rue Lhomond, 75231 Paris Cedex 05, France. \\ douglas@ihes.fr, c.m.hull@qmw.ac.uk .
}

We show that in certain superstring compactifications, gauge theories on noncommutative tori will naturally appear as D-brane world-volume theories. This gives strong evidence that they are well-defined quantum theories. It also gives a physical derivation of the identification proposed by Connes, Douglas and Schwarz of Matrix theory compactification on the noncommutative torus with $\mathrm{M}$ theory compactification with constant background three-form tensor field.

November 1997 
It is often stated that in toroidal compactification in superstring theory, there is a minimum radius for the torus, the string length. Tori with smaller radii can always be related to tori with larger radii by using T-duality.

However, this picture is not correct in the presence of other background fields, as is clear from the following simple example. Consider compactification on $T^{2}$ with a constant Neveu-Schwarz two-form field $B$. We quote the standard result [1]: under simultaneous T-duality of all coordinates, the combination $(G+B)_{i j}$ is inverted, where $G$ is the metric expressed in string units. Consider a square torus and take the limit

$$
R_{1}=R_{2} \rightarrow 0 ; \quad B \neq 0 \text { fixed }
$$

The T-dual torus has

$$
G+B=\frac{1}{R_{1}^{2} R_{2}^{2}+B^{2}}\left(\begin{array}{cc}
R_{2}^{2} & -B \\
B & R_{1}^{2}
\end{array}\right)
$$

and the T-dual radii go to zero as $\tilde{R}_{1}=\tilde{R}_{2} \sim R_{1} / B$.

Of course the full T-duality group is larger and includes an $S L(2, \mathbb{Z})$ acting on the upper half plane $B+i R_{1} R_{2}$ in the usual way. For any point there exists an $S L(2, \mathbb{Z})$ transformation which brings it to the fundamental region with $R_{1} R_{2} \geq \sqrt{3} / 2$, so that it would appear that there is again a minimal length. However, the particular $S L(2, \mathbb{Z})$ transformation that maps back to the standard fundamental region depends on $R_{1} R_{2}$ and varies wildly as we take the limit (何. In any situation involving objects which transform under $S L(2, \mathbb{Z})$, the physics of the limit is not well described by making these transformations.

A better way to get at the limit is to T-dualize a single dimension. This exchanges the complexified Kähler form $B+i R_{1} R_{2}$ and the complex structure of the torus $\tau=R_{2} / R_{1}$, to produce a theory with fixed volume and $B=0$, while the torus becomes highly anisotropic: $\tau^{\prime}=B+i R_{1} R_{2}$. Let us think of this torus as $\mathbb{R}^{2}$ quotiented by the two translations $x \rightarrow x+1$ and $x \rightarrow x+\tau^{\prime}$. In the limit $R_{1} R_{2} \rightarrow 0$, the original $T^{2}$ appears to degenerate to $\mathbb{R} \times S^{1}$ with coordinate $0 \leq x^{1}<1$ quotiented by an additional translation $x^{1} \rightarrow x^{1}+B$. The nature of such a quotient depends radically on whether $B$ is rational or irrational; if we interpret it in the latter case naively as a pointwise identification, it will not lead to a conventional Hausdorff manifold.

This is exactly the situation which was the original motivation for noncommutative geometry [2], leading us to try to interpret the resulting space as a noncommutative space. Not only is this a sensible thing to do, we will now give a physical argument which leads to gauge theory on the noncommutative torus as defined in [3]. 
Consider a theory of $N$ D0-branes on the original torus. After T-dualizing one dimension, this becomes a theory of $N$ D1-branes stretching along the T-dualed dimension; let them be extended in $x^{1}$, and placed at $x^{2}=0$. The compactness of $x^{2}$ will be implemented by placing images of the D1-branes at $x+n R_{2}$ for all integers $n$; in other words extended in $x^{1}$ and at $x^{2}=n R_{2} \cdot *$

This is not a conventional $U(N) 1+1$-dimensional gauge theory, because it contains additional light states. A string winding about $x^{2}$ will take the shortest path between a D1-brane and its image consistent with the boundary conditions on the D1-brane, Dirichlet in $x^{2}$ and Neumann in $x^{1}$. This means that the string will end at a right angle and follow a path $\left(x^{1}, s\right)$ for $0 \leq s<R_{2}$. Its length is $R_{2}$ and it is light, with mass $m \propto R_{2}$, so it must be kept in the low energy theory.

A string winding $w_{2}$ times will have mass $m \propto w_{2} R_{2}$, so we must consider fields with arbitrary dependence both on $x^{1}$ and integral $w_{2}$ to get the complete low energy spectrum. By the usual Fourier transform of $w_{2}$ to $x^{2}$, we could rewrite this as a $U(N)$ $2+1$-dimensional gauge theory, in terms of fields $A_{i}\left(x^{1}, x^{2}\right)$.

However, this is not a conventional $U(N) 2+1$-dimensional gauge theory either, because the open string which starts at $\left(x^{1}, 0\right)$ will end at $\left(x^{1}, w_{2} R_{2}\right)$ which is identified with $\left(x^{1}-w_{2} B / R_{1}, 0\right)$. The degrees of freedom do not live at points in $2+1$-dimensional space and thus the theory is not local in the conventional sense.

Still, it is not hard to guess what modification of the gauge theory is induced by non-zero $B$. When two open strings interact, the end of the first must coincide with the beginning of the second. We can express this in the $\left(x^{1}, w_{2}\right)$ basis by an interaction of the form

$$
\begin{aligned}
S_{i n t} & =\sum_{w_{2}, w^{\prime} 2} \int d x^{1} \phi_{1}\left(x^{1},-w_{2}-w_{2}^{\prime}\right) \phi_{2}\left(x^{1}, w_{2}\right) \phi_{3}\left(x^{1}-B w_{2} / R_{1}, w_{2}^{\prime}\right) \\
& =\sum_{w_{2}, w^{\prime}{ }_{2}} \int d x^{1} \phi_{1}\left(x^{1},-w_{2}-w_{2}^{\prime}\right) \phi_{2}\left(x^{1}, w_{2}\right) \exp \left(-\frac{B w_{2}}{R_{1}} \frac{\partial}{\partial x^{1}}\right) \phi_{3}\left(x^{1}, w_{2}^{\prime}\right) .
\end{aligned}
$$

We then Fourier transform, and go to coordinates of unit radius $\sigma_{i} \equiv R_{i} x^{i}$. The interaction becomes

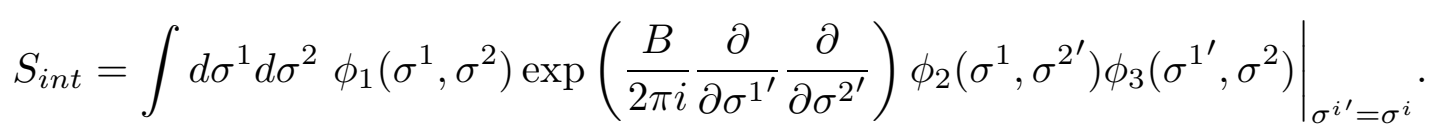

\footnotetext{
* The periodicities after $\mathrm{T}$ duality are $\sqrt{V^{\prime} / \Im \tau^{\prime}}=1 / R_{1}$ and $\sqrt{V^{\prime} \Im \tau^{\prime}}=R_{2}$.
} 
But this is exactly the characteristic interaction term in gauge theory on the noncommutative torus! Without repeating all the details (given in [4] and other works on this theory), the general connection on the noncommutative torus is a sum $\nabla_{i}+A_{i}$ where $\nabla_{i}$ is a constant curvature connection and $A_{i}$ are are elements of the algebra defined by

$$
Z^{2} Z^{1}=e^{2 \pi i B} Z^{1} Z^{2}
$$

One can be more concrete by choosing an identification $Z^{i} \sim e^{2 \pi i \sigma^{i}}$; then $\nabla_{i}=\partial / \partial \sigma^{i}$ and the noncommutative multiplication is exactly realized by the differential operator which appears in (2). Since the interactions are all associated with the end of one open string joining the beginning of another (as is clear by the Chan-Paton rules and the fact that the resulting action is a single trace), every time two fields appear multiplied in the D-brane action, such an operator will appear. This is exactly the action of gauge theory on the noncommutative torus.

The essential phenomenon can already be seen in the D0-brane theory without Tduality. Consider a state containing an open string starting on D0-brane $i$, winding $w_{1}$ times around $x^{1}$ and ending on brane $j$. If one carries it about the loop $x^{2}$, it will pick up a phase $e^{2 \pi i B}$. Thus its interactions with the strings starting on $j$, winding $w_{2}$ times around $x^{2}$ and ending on $i$ must contain a relative phase $e^{2 \pi i B w_{1} w_{2}}$ between the case of attaching at $i$ and attaching at $j$.

To come back to the original issue of minimal length, we have found that after dualizing to a description with $B=0$, the radii of the dual torus on which the final $2+1$-dimensional theory is defined are $1 / R_{1}$ and $1 / R_{2}$, and do become large in the limit.

A context in which the nature of the limit (1) is important is the recent proposal by Sen and by Seiberg [5] for a general definition of Matrix theory [6,7]. They propose quite generally that Matrix theory definitions of M theory and DLCQ M theory (with a compact null direction) can be obtained from type IIa superstring backgrounds by considering a sector with D0-branes and taking a scaling limit, which includes the limit $R_{1}, R_{2} \rightarrow 0$.

Recently in [4] it was proposed that DLCQ M theory backgrounds with non-zero background three-form $C_{-i j}$ along the null direction have Matrix theory definitions obtained by replacing conventional gauge theory with gauge theory on the noncommuting torus. By the standards of superstring duality, quite strong evidence (equivalence of the BPS mass formulas on both sides) was given. On the other hand gauge theory on the noncommuting torus has been unjustly neglected by physicists and basic questions such as whether 
it is a well-defined quantum theory have not yet been answered, leaving some room for scepticism.

Applying the idea of [5], we can define Matrix theory in these backgrounds by starting with type IIa superstring theory with non-zero $B_{i j}$ field and taking a scaling limit. This corresponds to $\mathrm{M}$ theory $\int d^{11} x C_{11, i j}$ which under a boost turns directly into the background of interest. Thus the result we just described leads directly to matrix theory on the noncommutative torus as proposed in [4].

We see that gauge theory on the noncommutative torus is a well-defined quantum theory, considered as a subsector of string theory. This argument might well prove that its low energy limit decoupled from string theory makes sense as a renormalizable quantum theory. The only caveat is that the non-locality of the theory might invalidate the standard effective field theory arguments. Since these are concrete theories with an action and a conventional perturbative expansion, this question can be studied using standard techniques, and we look forward to a detailed investigation of this point. 


\section{References}

[1] A. Giveon, M. Porrati and E. Rabinovici, Phys.Rept. 244 (1994) 77-202, hepth/9401139; and references there.

[2] A. Connes, Noncommutative Geometry, Academic Press, 1994.

[3] A. Connes, C. R. Acad. Sci. Paris Sér. A-B 290 (1980) A599-A604;

M. Pimsner and D. Voiculescu, J. Operator Theory 4 (1980) 93-118;

A. Connes and M. Rieffel, "Yang-Mills for noncommutative two-tori," in Operator Algebras and Mathematical Physics (Iowa City, Iowa, 1985), pp. 237-266, Contemp. Math. Oper. Algebra. Math. Phys. 62, AMS 1987;

M. Rieffel, "Projective modules over higher-dimensional non-commutative tori," Can. J. Math, 40 (1988) 257-338.

[4] A. Connes, M. R. Douglas and A. Schwarz, "Noncommutative Geometry and Matrix Theory: Compactification on Tori," hep-th/9711162.

[5] A. Sen, "D0 Branes on $T^{n}$ and Matrix Theory," hep-th/9709220;

N. Seiberg, "Why is the Matrix Model Correct?" hep-th/9710009.

[6] T. Banks, W. Fischler, S. H. Shenker and L. Susskind, Phys. Rev. D55 (1997) 51125128; hep-th/9610043.

[7] L. Susskind, hep-th/9704080. 